\title{
Molecular and biochemical responses in the midgut of the silkworm, Bombyx mori, infected with Nosema bombycis
}

\author{
Zhi Li', Yu Wang ${ }^{1}$, Linling Wang ${ }^{1 *}$ and Zeyang Zhou ${ }^{1,2}$
}

\begin{abstract}
Background: Microsporidia are a group of eukaryotic intracellular parasites that infect almost all vertebrates and invertebrates. However, there is little information available of how microsporidia obtain nutrients and energy from host cells. The purpose of this study was to investigate the energy and material requirements of Nosema bombycis for the invasion procedure through analyzing the global variation of the gene expression, protein abundance, fatty acids level and ATP flux induced by the microsporidia N. bombycis infection in the midgut of the silkworm Bombyx mori.

Methods: A suppression subtractive hybridization (SSH) and quantitative real-time PCR (qPCR) analysis were performed to identify the genes upregulated in the midgut of B. mori $48 \mathrm{~h}$ following N. bombycis infection. Gene Ontology (GO) and the Kyoto Encyclopedia of Genes and Genomes (KEGG) analyses were used to annotate and summarize the differentially expressed genes, according to the categories 'molecular function', 'cellular component' and 'biological process'. To evaluate the nutrition material and energy costs in B.mori infected by N. bombycis, biochemical analysis was performed to determine the variation of protein abundance, fatty acid levels and ATP flux with or without the microsporidia N. bombycis infection in the midgut of the silkworm B. mori.
\end{abstract}

Results: A total of 744 clones were obtained, 288 clones were randomly selected for sequencing, and 110 unigenes were generated. Amongst these, 49.21\%,30.16\% and 14.29\% genes were involved in 19 molecular functions, 19 biological processes and nine cellular components, respectively. A total of 11 oxidative phosphorylation- and eight proton-coupled ATP synthesis-related genes were upregulated. Seven protein degradation-, three fat degradationrelated genes were upregulated, and no genes related to the de novo synthesis of amino acids and fatty acids were significantly upregulated. The data from the biochemical analysis showed the contents of total protein and ATP of B. mori midgut tissues decreased significantly, whereas the fatty acid content did not significantly change after four days of $N$. bombycis infection. Microsporidia N. bombycis infection upregulated the expression level of genes involved in host ATP synthesis, protein and fat degradation, which eventually causes the obvious decline of protein content and ATP synthesis in the host midgut, whereas the fatty acids content did not change significantly.

Conclusions: This study suggested to some extent that $N$. bombycis invasion can activate the host protein degradation and accelerate the production of host ATP. Microsporidia of N. bombycis show preference for proteins rather than fatty acids from the host to ensure the material preparation required by their parasitic life-cycle. Requirements of $\mathrm{N}$. bombycis for energy were also mainly dependent on the host ATP production. This study provides a new data that may help our understanding of the molecular mechanisms of obtaining energy and nutrients from the host by the microsporidium N. bombycis.

Keywords: Nosema bombycis, Silkworm midgut, Interaction, Differential gene expression, Biochemical response

\footnotetext{
* Correspondence: wanglinling2005@yahoo.com

${ }^{1}$ College of Life Sciences, Chongqing Normal University, Chongqing 401331,

China

Full list of author information is available at the end of the article
} 


\section{Background}

Microsporidia are a group of eukaryotic intracellular parasites that infect almost all vertebrates and invertebrates and cause serious human diseases and major economic losses in the livestock industry. There are no prospective drugs to counteract this pathogen. The pébrine disease caused by microsporidia Nosema bombycis infection is a devastating disease for sericulture. Nosema bombycis is one of the major quarantine pests that have been established in silk-producing countries [1]. This microsporidian has evolved a unique way to invade the host cell. First, upon appropriate environmental stimulation, $N$. bombycis extrudes the polar tube, penetrates the membrane of the host cell and enters the cytoplasm. Then, their sporoplasm is transferred into the cytoplasm of the host cells, where the spores develop and complete the life-cycle [2-4]. This injection-like method can effectively circumvent the attacks from the host's multiple defence systems. Similar to the other reported microsporidia, the genome of the obligate parasite $N$. bombycis is small and compact [5-8]. The parasites lack mitochondria and only contain a mitosome which is thought to be a relic of the mitochondria; some genes involved in energy and material metabolism have been lost $[5,9,10]$. Their production of energy and material is far from enough to facilitate the growth and reproduction. Much energy must be obtained from the host cells.

The silkworm Bombyx mori is the natural host of $N$. bombycis. The mechanism of the interaction between $B$. mori and its pathogens has attracted considerable attention [11-13]. Over the past few years, researchers have used Suppression Subtractive Hybridization (SSH), DNA microarray system, high throughput expressed sequence tags, and isobaric Tag for Relative and Absolute Quantization (iTRAQ) proteomics-based methods to examine changes in gene transcription in the midgut of the silkworm B. mori, mainly focusing on the host immune responses to exogenous pathogenic infection [14-19]. As the first directly infected organ, the midgut has an induced high level of tissue-specific gene expression. Previous research found that a total of 36 genes and 20 novel expressed sequence tags (ESTs) were altered in the midgut of $B$. mori infected by $B$. mori cytoplasmic polyhedrosis virus (BmCPV) [14]. Recently, iTRAQ-based quantitative proteomic analysis found that a putative $\mathrm{p} 62 /$ sequestosome-1 protein in silkworm was upregulated and may play an important role in regulating the autophagy and apoptosis (especially apoptosis) after BmCPV infection [15]. In addition to the BmCPV, the latest research based on comparative subcellular proteomics analysis found that 16 proteins in larval silkworm midgut were potentially involved in repressing $B$. mori nucleopolyhedrovirus (BmNPV) infection [16]. Moreover, BmNPV invasion also causes complex protein alterations in the larval midgut, and these changes are related to the cytoskeleton, immune response, apoptosis, ubiquitination, translation, ion transport, endocytosis and endopeptidase activity [17]. In addition, pathogenic Bacillus bombyseptieus invasion can also induce a strong transcriptional response in the midgut, with altered transcription of genes encoding metalloproteinases, hydrolases, lipases and chitin structural proteins [18]. For pathogenic microsporidia, the only study of transcriptional responses of $B$. mori to microsporidia $N$. bombycis infection was published in 2013 [19]. Many immunerelated genes involved in melanization, humoral and cellular immunity exhibited an apparently induced upregulated expression. However, some genes involved in the energy and material transport between $B$. mori and the pathogenic $N$. bombycis remain to be further addressed. So far, there is little information available of how pathogens such as $N$. bombycis obtain nutrients and energy from their host $B$. mori. The only indirect evidence showed that in vitro spore germination upregulated the expression level of some genes involved in the glycolysis, the pentose phosphate pathway, purine and pyrimidine metabolism, suggesting preparations of energy generation and substance synthesis for $N$. bombycis following invasion and proliferation inside the host [20].

In this study, the global variation of the gene expression, protein abundance, fatty acids level and ATP flux induced by the microsporidia $N$. bombycis infection in the midgut of the silkworm $B$. mori was investigated. Our work provides new insights into the $N$. bombycis metabolic requirements and the interaction between microsporidia and their insect host and lists candidate drug targets for the treatment of microsporidian diseases of the silkworm B. mori.

\section{Methods \\ Infection protocol}

The B. mori strain used in this study (Dazao P50) was preserved in the Southwest University Silkworm Gene Library. The $N$. bombycis CQ1 isolates were obtained from the State Key Laboratory of Silkworm Genome Biology in Southwest University and preserved by the China Veterinary Culture Collection Center (CCVC) (Accession no. CCVC102059).

Bombyx mori larvae were reared under conventional conditions with mulberry leaves [21]. The larvae were then randomly divided into 2 groups, with the experimental group being fed $5 \mu \mathrm{l}$. bombycis mature spores (suspended at $1 \times 10^{6}$ spores $/ \mathrm{ml}$ ) sprayed on the mulberry leaves; the control group was fed $5 \mu$ l sterile water. After $48 \mathrm{~h}$, midgut tissues of 3 larvae from both groups were isolated, snap-frozen in liquid nitrogen, and preserved at $-80{ }^{\circ} \mathrm{C}$. The remaining larvae from each group were reared until the 4th day after infection, 
when the midgut was isolated for electron microscopy and the determination of the total protein and fatty acids.

\section{RNA isolation and CDNA preparation}

Total RNA of the midguts of $N$. bombycis-infected larvae at $48 \mathrm{~h}$ was extracted using the RNAiso Plus kit (Takara BioTech, Dalian, China). Double-stranded cDNA was synthesized by using the SMARTer cDNA Synthesis kit (Clontech, Shanghai, China) and purified by using the CHROMA-SPIN-1000 chromatography column (Clontech). All procedures were carried out according to manufacturers' instructions.

\section{Rsal digestion and purification of double-stranded cDNA}

The purified double-stranded cDNA was digested with RsaI enzyme in RsaI restriction buffer at $37{ }^{\circ} \mathrm{C}$ for $3 \mathrm{~h}$, and the reaction was terminated by adding $0.2 \mathrm{M}$ EDTA. The digested cDNA was purified by phenol-chloroform extraction method and dissolved in double-distilled water. The final cDNA concentration was adjusted to $300 \mathrm{ng} / \mu \mathrm{l}$ for subsequent experimental work.

\section{Construction of subtracted cDNA libraries}

Subtractive hybridization was performed for the midgut cDNA products with the experimental group as the tester and the control group as the driver, followed by nested PCR. The efficiency of subtractive hybridization was analyzed based on changes in the abundance of the housekeeping gene G3PDH before and after the subtraction. The Takara DNA Fragment Purification kit (Takara BioTech, Dalian, China) was used to purify the PCR products; these were ligated with pMD19-T vector by using the Takara DNA Ligation kit (Takara BioTech, Dalian, China). The ligation product was transformed into Escherichia coli competent cells JMl09 (Takara BioTech, Dalian, China), spread on LB plates, and cultured overnight at $37^{\circ} \mathrm{C}$. The numbers of both blue and white colonies were counted, and 24 white colonies with $1 \mathrm{~mm}$ diameter from one plate were randomly selected and collected into bacteria-detection PCR tubes as templates. The PCR reactions were carried out with the universal primers targeting both ends of the T vector, M13-47 and RV-M to determine the success of the ligation and the size of the inserted fragment.

\section{Sequencing and bioinformatics analysis}

A total of 288 positive clones with inserted fragments were selected for plasmid extraction (Takara BioTech) and sequencing (Invitrogen BioTech, Shanghai, China). The assembled sequences were analyzed by BLAST program. Other sequences from NCBI (http://www.ncbi.nlm. nih.gov/) and the silkworm genome database (http:// www.silkdb.org/silkdb/) were used to identify nucleic acid homologous. Gene ontology (GO) annotation was performed for the differentially expressed genes using the Blast2GO software and the online molecular annotation system Capital Bio Corp (MAS 2.0, http:// www.capitalbio.com/). Upregulated genes were further functionally analyzed using the online metabolism pathway database, the Kyoto Encyclopedia of Genes and Genomes (KEGG) (http://www.genome.jp/kegg/).

\section{Quantitative real-time PCR analysis}

Midgut total RNA was extracted using the RNasy Protect Mini Kit (Qiagen, Shanghai, China). The RNA was diluted to $50 \mathrm{ng} / \mu \mathrm{l}$ for cDNA synthesis using the PrimeScriptTM RT cDNA reagent Kit (Takara BioTech). The quality of cDNA product was evaluated by gel electrophoresis. qPCR was carried out with SYBR Premix EX Taq II (Takara BioTech) in an iQ5 qPCR machine using $\beta$-actin as the reference gene and the primers listed in Table 1 . The PCR products were determined as specific when the melting curves were single peaks. qPCR was performed for a series of dilution standards of 7 genes, and the amplification efficiency of these genes was close to $100 \%$. qPCR reaction was repeated three times for each sample, and the mean of the three repeats was compared to the amplification of $\beta$-actin for quantification. qPCR products were sequenced by Shanghai Biological Engineering Technology Services Co., Ltd. to confirm whether the amplified fragments were the target sequences. For the analysis of the data and graphical representation, the $2^{-\Delta \Delta \mathrm{Ct}}$ method was used [22]

\section{Protein, fatty acid and ATP analysis of the midgut tissue}

For protein concentration analysis, after 4 days of $N$. bombycis infection, the midgut tissues of $B$. mori larvae from infection and control groups were isolated, weighed, homogenized in the RIPA lysis buffer $(50 \mathrm{mM}$ Tris, $150 \mathrm{mM} \mathrm{NaCl}, 1 \%$ Triton $\mathrm{X}-100,1 \%$ sodium deoxycholate, $0.1 \%$ SDS, sodium orthovanadate, sodium fluoride, EDTA, $1 \mathrm{mM} \mathrm{PMSF)}$ and centrifuged at $12,000 \times g$ and $4{ }^{\circ} \mathrm{C}$ for $10 \mathrm{~min}$. The supernatant was collected to determine the protein content using the classical Bradford method [23] and the Bradford Protein Concentration Kit (Beyotime, Shanghai, China), according to the manufacturer's instructions.

For analysis of fatty acids, the pellet was dried, weighed, resuspended in chloroform - methanol - sterile $\mathrm{H}_{2} \mathrm{O} \quad(6: 12: 5, \mathrm{v} / \mathrm{v} / \mathrm{v})$ and rotated for $14 \mathrm{~h}$ at room temperature. Then, chloroform - sterile $\mathrm{H}_{2} \mathrm{O}(6: 6, \mathrm{v} / \mathrm{v})$ was added, mixed rapidly and filtered. The chloroform layer was dissolved in $\mathrm{N}$-hexane and $\mathrm{KOH}$ - methanol solvent $(0.5 \mathrm{~mol} / \mathrm{l})$ with $4: 1$ volume ratio at $70{ }^{\circ} \mathrm{C}$ for 20 min, vibrated in the ultrasonic cleaner for $5 \mathrm{~min}$, centrifuged at $3500 \times g$ for $10 \mathrm{~min}$, and the fatty acids were collected from the N-hexane layer. Finally, quantitative 
Table 1 Primers used in real-time PCR

\begin{tabular}{llll}
\hline Target gene & GenBank ID & Forward primer (5'-3') & Reverse primer (5'-3') \\
\hline G3PDH & DQ443421.1 & CATATTAAGCTACCGTCAGA & GGAAACCAATCTTGCCGTGT \\
B-actin & BMU49854 & GGATGTCCACGTCGCACTTCA & CGGAGAGGTCGCGTCCAAACA \\
Serine protease 1 & NP_001036826 & GCGGCCGAGGTACCAGGATT & CGGAGCGGGTGTTGGTCAGT \\
Serine protease 5 & AAX39408 & TGGTGCTCACCCCCATCTTGC & CGGCCGAGGTACCAAAAGCGA \\
Serine protease 11 & AAB26023.1 & TCGCGACCACCGACGTCCAA & TGGGGTGCTACATCGCTCGGA \\
Lipase & NP_001036966 & GGTACATGGGGACGATTGCGAGC & GAACACCCTCGCCATCAGGCAT \\
Membrane protein & ABK23569 & CGCTAGCGCCAGTGGACGTG & CGGAGAGGTCGCGTCCAAACA \\
Vacuolar ATP synthase subunit G & NP_001040287 & GGCAGGTTTCTTGGAGCCGA & CGGGCAGGTACCGAGCTGAT \\
\hline
\end{tabular}

fatty acid analysis was conducted by gas chromatography with mass spectrometric detection (GC-MS, Thermo Trace, Silicon Valley, USA) [24], with minor modifications. Briefly, the oven temperature was set to $40{ }^{\circ} \mathrm{C}$ for $1 \mathrm{~min}$ and then increased to $260{ }^{\circ} \mathrm{C}$ at a rate of $5{ }^{\circ} \mathrm{C} / \mathrm{min}$ and holding for $10 \mathrm{~min}$. The helium carrier gas was set at a flow rate of $1 \mathrm{ml} / \mathrm{min}$. The GC inlet was held at a temperature of $230{ }^{\circ} \mathrm{C}$. The equilibration time between injections was $0.2 \mathrm{~min}$. The temperatures of the MS source and the MS quadrupole were maintained at $250^{\circ}$ $\mathrm{C}$ and $200{ }^{\circ} \mathrm{C}$, respectively. The mass spectra were acquired in full scan mode over the mass range of 50-500 $\mathrm{m} / \mathrm{z}$. Finally, fatty acid relative content was calculated according to the peak areas by integrating the respectively extracted ion chromatograms (EIC) with Xcalibur software.

Adenosine triphosphate (ATP) content in the midgut tissues was measured using a firefly luciferase-based ATP assay kit S0026 (Beyotime), according to the manufacturer's protocol. Midgut tissues were collected after washing in PBS and lysed in $200 \mu \mathrm{l}$ of lysis buffer $/ 20 \mathrm{mg}$ tissue, then homogenated with a glass homogenizer. The lysates were centrifuged at $12,000 \times g$ for $5 \mathrm{~min}$ at $4{ }^{\circ} \mathrm{C}$. The supernatants were collected and $20 \mu \mathrm{l}$ was added to a 96-well plate with $100 \mu \mathrm{l}$ pre-added ATP test solution followed by incubation at RT for $10 \mathrm{~min}$. OD values were measured at $570 \mathrm{~nm}$ using a fluorescence multimode microplate reader (Tecan Infinite M200 Pro, Switzerland). The ATP content was calculated from the standard curve [25].

\section{Statistical analysis}

The average values of biological replicates $(n=3)$ were given as the mean \pm standard deviation (SE). Statistically significant differences between the infected and control non-infected for total protein, fatty acid and ATP content, and qRT-PCR determination was subjected to the Student's t-test in the Statistical Package for Social Science (SPSS version 23.0; SPSS Inc., Chicago, IL, USA). A Pvalue of $\leq 0.05$ was considered as statistically significant.

\section{Results}

\section{Confirmation of infection}

The midguts of $B$. mori larvae infected by $N$. bombycis for 4 days were isolated and observed under the electron microscope, which confirmed infection by $N$. bombycis compared with the control group. Also, five gene fragments identified by the subtractive library screening perfectly matched the $N$. bombycis genomic data (http:// silkworm.genomics.org.cn/), demonstrating the effectiveness of the infection.

\section{Quality of the subtracted cDNA libraries}

Subtractive hybridization was performed for the midgut cDNA products with the experimental group as the tester and the control group as the driver, followed by two rounds of PCR. Analysis of the PCR products showed that the enriched bands were detectable in the subtractive group, suggesting the effective enrichment of differentially expressed genes (Fig. 1a).

As shown in Fig. 1b, the PCR product of the housekeeping gene G3PDH (glyceraldehyde-3-phosphate dehydrogenase) could be observed after 18 cycles of amplification without subtractive hybridization. Following subtractive hybridization, the amplified G3PDH was barely observed even after 28 cycles of amplification, this confirming the efficiency of the subtraction protocol.

A total of 769 colonies were generated, 744 of which were white $(<5 \%$ self-ligation rate). Twenty-four white colonies were randomly selected for PCR analysis, and 21 of them contained inserted fragments $(87.5 \%$ positive colony rate). The fragment length ranged between $500-$ $1000 \mathrm{bp}$, with most of them within the range of 600-800 bp. A total of 288 clones were randomly selected for sequencing, and 265 positive sequences were obtained after eliminating low-quality and vector sequences.

\section{GO and KEGG analysis of differentially expressed genes}

The 265 positive sequences were aligned with BlastX, and repetitive sequences were removed. As a result, 110 sequences were annotated, 102 of which showed significant homology with genes encoding proteins with 


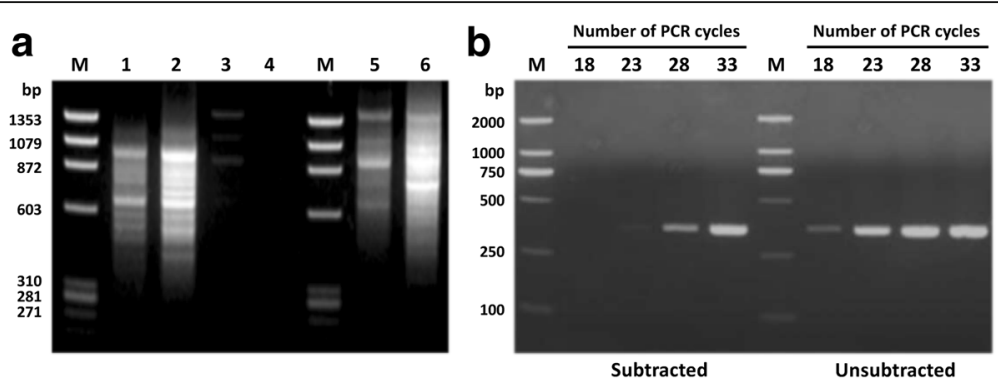

Fig. 1 PCR evaluation of subtractive hybridization efficiency. a Gel electrophoresis of subtractive hybridization products after two rounds of PCR amplification. Lane M: $\Phi$ X174-Hae III digest Marker; Lane 1: double PCR product of subtractive hybridization group; Lane 2: double PCR product of non-subtractive hybridization group; Lane 3: positive control; Lane 4: negative control; Lane 5: double PCR product of mouse control (subtractive hybridization); Lane 6: double PCR product of mouse control (no subtractive hybridization). b Gel electrophoresis of G3PDH gene PCR products using subtractive and non-subtractive cDNA as templates with 18,23, 28 and 33 cycles of amplification

known functions in the database (Additional file 1: Table S1). GO functional analysis revealed that $49.21 \%$ of the differentially expressed genes were involved in 19 molecular functions, along with $30.16 \%$ of them playing roles in 19 biological processes and $14.29 \%$ of them being involved in 9 cellular components (Fig. 2).

In level-2 GO classification, the differentially expressed genes were mainly involved in molecular function activity (Fig. 2a) as below: serine-type peptide chain endoproteinase activity, hydrogen transport ATP activity, hydrogen transport ATP synthase activity, and cytochrome $c$ oxidase activity, cytochrome $c$ oxidase activity, acyl-coenzyme A binding activity, trivalent iron-binding activity, GTP binding activity, endonuclease activity, and nucleotide binding and catalytic activity. The differentially expressed genes in biological processes (Fig. 2c) mainly were involved in proteolysis (16 genes, 27.78\%), protein synthesis (12 genes, 22\%), ATP synthesiscoupled proton transport (8 genes, 14.81\%), electron transport and ATP biosynthesis. The subcellular distribution pattern of the differentially expressed genes was also analyzed. As shown in Fig. 2b, the ribosomes (10 genes, 23.81\%), proton-transporting two-sector ATPase complex (8 genes, 19.5\%), intracellular (11 genes, $26.19 \%$ ) and membrane (7 genes, $16.67 \%$ ) were the main cellular components involved (Fig. 2b).

KEGG pathway analysis revealed that the differentially expressed genes were involved in fatty acid metabolism, polyketide sugar unit biosynthesis, oxidative phosphorylation, streptomycin biosynthesis, propionate metabolism, $\beta$-pyruvate metabolism, and degradation of valine, leucine, isoleucine, and isoleucine (Fig. 3). Relatively more genes were involved in metabolism, oxidative phosphorylation, mitochondria- or ubiquitin-mediated proteolysis and fatty acid metabolism.

\section{Quantitative real-time PCR}

To validate the changes in mRNA abundance detected by $\mathrm{SSH}$ and analyze the transcriptional responses of the silkworm to $N$. bombycis infection, qPCR was performed on 6 differentially expressed genes, including serine protease 1 (sp1, accession number NM_001043361), serine protease 5 (sp5, accession number AAX39408.1), serine protease 11 (sp11, accession number JX312360.1), lipase (lp, accession number XM_012695610.2), membrane protein ( $m p$, accession number XM_021353371.1), and vacuolar ATP synthase subunit G (vas, accession number NM_001046822.1). As shown in Fig. 4, these genes were significantly upregulated 48 hours after $N$. bombycis infection except for sp11 at 24 hours, consistent with the results from the subtracted cDNA libraries-based investigation (Additional file 1: Table S1). The results of qPCR further demonstrated that the subtractive hybridization library was successfully constructed and also suggested that these genes may be involved in the interaction between $N$. bombycis and the host $B$. mori.

Total protein, fatty acid and ATP contents in midgut tissue To evaluate whether nutrients materials and energy in the midgut of $B$. mori change in response to $N$. bombycis infection, the content of protein, fatty acid and ATP in the midgut tissues was determined. GC-MS analysis results showed that the fatty acids in $B$. mori midgut mainly consisted in $\mathrm{C}_{17} \mathrm{H}_{34} \mathrm{O}_{2}$ (hexadecanoic acid methyl esters), $\mathrm{C}_{18} \mathrm{H}_{28} \mathrm{O}_{3}$ (benzenepropanoic acid, 3,5-bis(1,1dimethylethyl)-4-hydroxy-, methyl ester), $\mathrm{C}_{18} \mathrm{H}_{36} \mathrm{O}_{2}$ (palmitic acid ethyl ester), $\mathrm{C}_{19} \mathrm{H}_{38} \mathrm{O}_{2}$ (methyl stearate), and $\mathrm{C}_{20} \mathrm{H}_{40} \mathrm{O}_{2}$ (octadecanoic acid ethyl ester) (Fig. 5b, Additional file 2: Table S2). No significant changes in fatty acids content after 4 days of $N$. bombycis infection were observed compared with the control non-infected groups (Fig. 5b). However, it is worth noting that the total protein and ATP contents were significantly decreased due to $N$. bombycis infection (Fig. 5a, c).

\section{Discussion}

The complex interaction between pathogens and insects begins in the midgut cells [26, 27]. Many studies have 

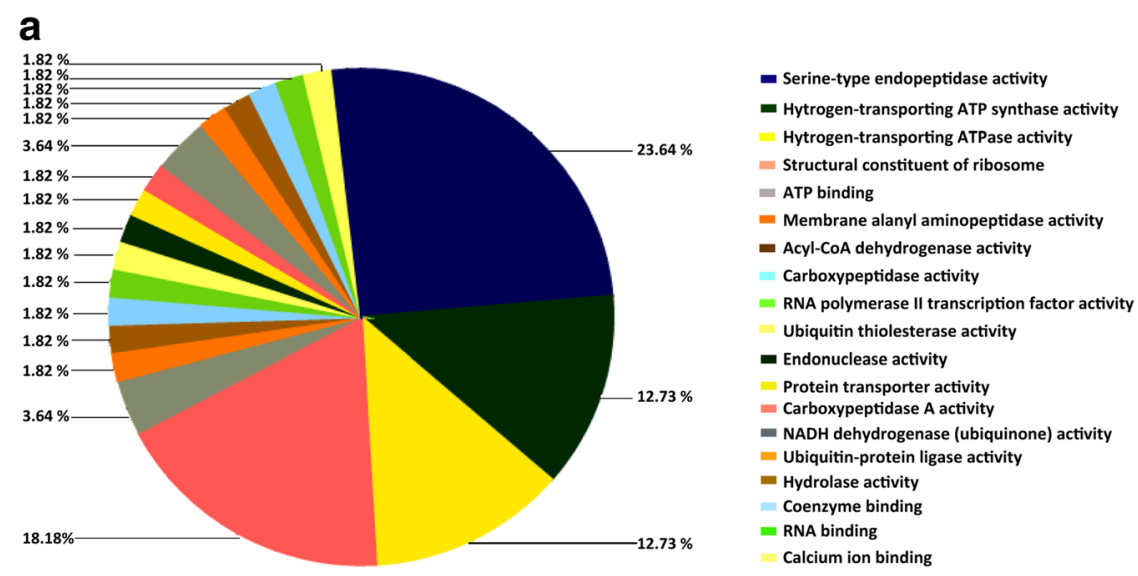

\section{b}

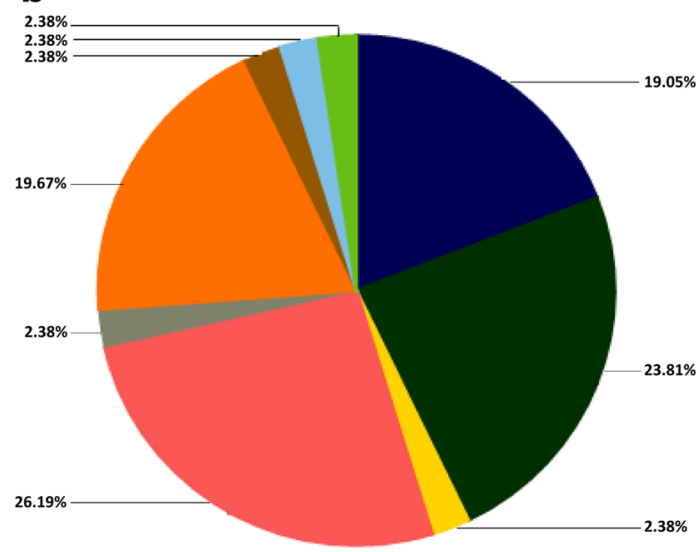

- Proton-transporting two-sector ATPase complex

- Ribosome

- Transcription factor TFIIF complex

- Intracellular

- Microsome

- Membrane

- Ribonucleoprotein complex

- Integral to membrane

Cytoplasm

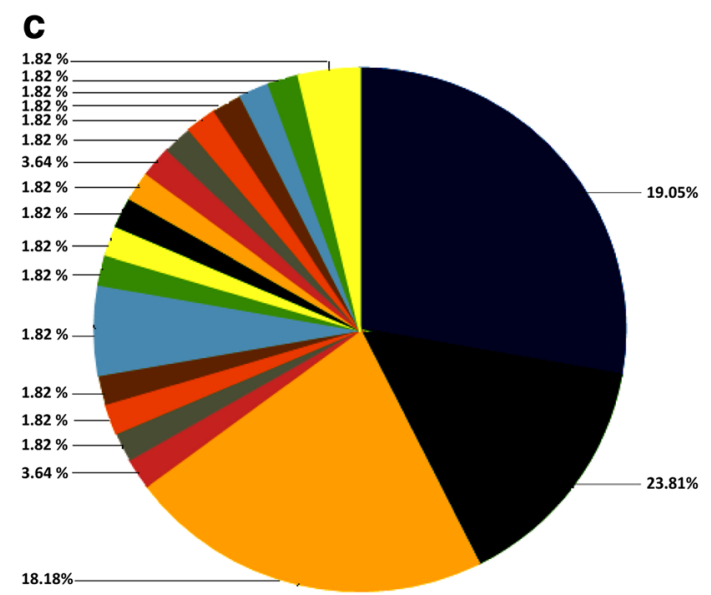

- Proteolysis

- ATP synthesis coupled proton transport

- Protein biosynthesis

- Dicarboxylic acid transport

- Response to toxin

- Transcription initiation from RNA polymerase II protor

- Iron ion homeostasis

Electron transport

- Mitochondrial electron transport, NADH to ubqinone

Peptidoglycan catabolism

- mRNA metabolism

- Ubiquitin cycle

- Ubiquitin-development protein catabolism

- ATP biosynthesis

- Intracellular protein transport

- Protein modification

- Transport

- Cellular metabolism

Fig. 2 Distribution of Gene Ontology (GO) functional categories (level 2) of Suppression Subtractive Hybridization libraries for Bombyx mori. a Molecular function. b Cellular component. c Biological process. Each annotated sequence is assigned at least one GO term. Numbers refer to percentage of assigned unigenes in each category

observed that pathogenic microsporidia N. bombycis, cytoplasmic polyhedrosis virus (CPV), Bombyx mori nucleopolyhedro virus (BmNPV), Escherichia coli, Bacillus bombyseptieus, Beauveria bassiana and Bacillus thuringiensis infections promote the expression of immune-related genes [11-13]. However, there is little information available of how microsporidia obtain the material and energy from host cells. The microsporidia successfully enter the host midgut cells where the spores initiate the first-round proliferation. In this stage, similar to other intracellular pathogens, the spores need nutrients and energy from host cells to meet for proliferation. 


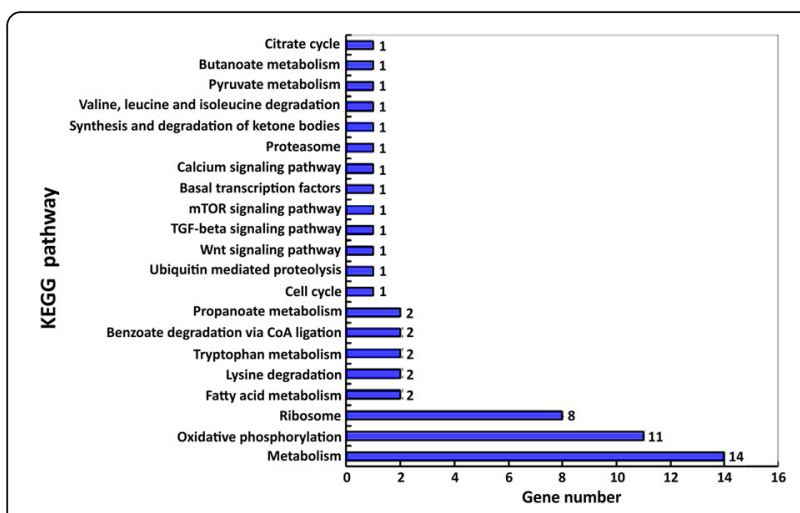

Fig. 3 KEGG classification of non-redundant unigenes for Bombyx mori Suppression Subtractive Hybridization libraries

However, since microsporidia lack mitochondria, and their tricarboxylic acid (TCA) cycle and oxidative phosphorylation pathways for ATP production have been lost $[2,5,9,10,26-28]$, the energy generated by the glycolysis pathway is insufficient to facilitate their growth and reproduction. Therefore, additional energy needs to be obtained from the host cells. One study speculated that microsporidia Encephalitozoon cuniculi might use NTT proteins to exploit the ATP pool of its eukaryotic host to complete their life-cycle [29].

In this study, we performed SSH and qPCR to investigate gene expression patterns in the midgut of $B$. mori infected with the microsporidian $N$. bombysis and paid particular attention to genes related to energy metabolism, fat and protein degradation. SSH analysis showed that eight genes related to proton-coupled ATP synthesis and 11 genes involved in oxidative phosphorylation of $B$. mori were significantly upregulated after $48 \mathrm{~h}$ of $N$. bombycis infection. These results indicate that $N$. bombycis invasion resulted in upregulation of the expression levels of host genes related to ATP synthesis, which might significantly increase ATP production to ensure $N$. bombycis energy requirements. Interestingly, in this study, $\mathrm{SSH}$ analysis indicated that the expression level of succinyl coenzyme A synthetase (XM_004928937.3), a key enzyme in the TCA cycle of the host midgut, was significantly increased, suggesting that ATP production in the host was enhanced through the TCA cycle [30, 31]. However, biochemical analysis indicated that the ATP content in the infected midgut tissue did not significantly increase as expected. Instead, the amount of ATP content was significantly decreased compared with the uninfected midgut. Given the expression level of those ATP synthesis related genes in host midgut were significantly upregulated (Fig 2a), this result implied the existence of an additional energy consumer during this procedure. The $N$. bombycis might act this role elevating the total energy expenditure and sharing the increased ATP sythesized by $B$. mori, which is required by the extra energy demand of its specific parasitic life.

As an obligate intracellular parasite with a highly reduced and compacted genome, the microsporidian $N$. bombycis has lost some genes involved in material metabolism [32, 33]. For example, $N$. bombycis lacks a large number of genes related to the de novo synthesis of amino acids [6]. Most intracellular pathogens utilize fatty acids as carbon sources to cope with nutrient deprivation during the infection process, and the microsporidian $N$. bombycis may be no exception. Nosema bombycis may need protein and fatty acids from the host to ensure the completion of the life-cycle. Interestingly, in this study, transcriptome data showed that protein degradation related genes in $B$. mori were significantly upregulated following $N$. bombycis invasion. The ubiquitin-proteasome system (UPS) is the major intracellular protein degradation pathway. Nosema bombycis infection leads to the elevated expression of protein degradation related enzyme in the host midgut, including the $\mathrm{S}$-phase kinase-associated protein 1 (NP_001040518.1) and the ubiquitin-conjugating enzyme E2G (XP_973689.1) both of which are involved in the ubiquitin-proteasome pathway. In addition, serine
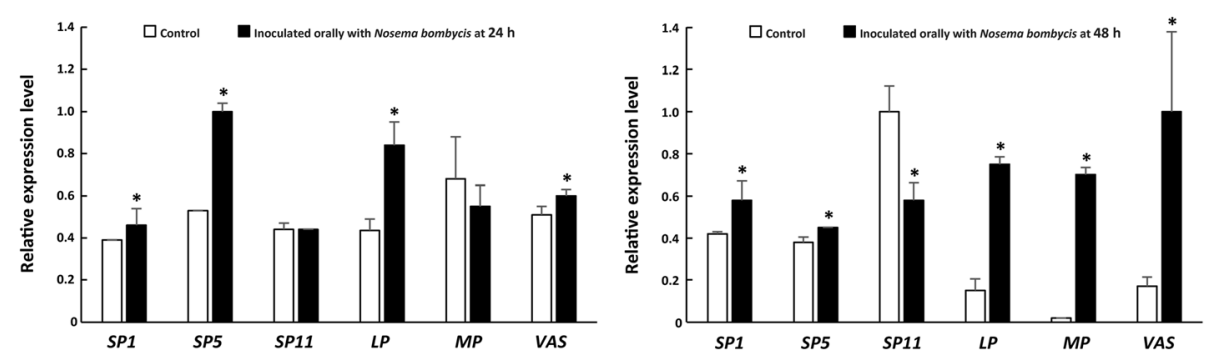

Fig. 4 Quantitative real-time PCR analysis for six genes expressed in the midgut of Bombyx mori. The expression levels of selected genes were upregulated at the time points of 24 and 48 hours in the midgut of Bombyx mori infected by Nosema bombycis. Abbreviations: sp1: serine protease 1; sp5: serine protease 5; sp11: serine protease 11; Ip: lipase; mp: membrane protein; vas: vacuolar ATP synthase subunit G. Data are presented as the mean \pm SE of triplicate experiments. Statistically significant differences between the infected groups and control non-infected groups were determined using Student's t-test. ${ }^{*}$-value of $\leq 0.05$ was considered as statistically significant 

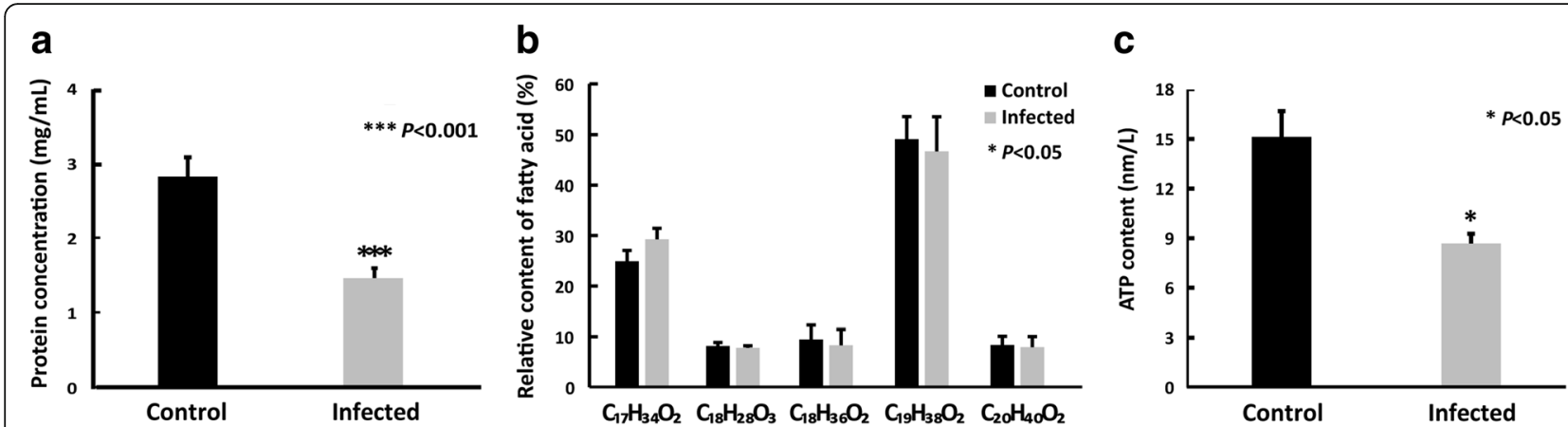

Fig. 5 Analysis of the total protein, fatty acid and ATP contents in the midgut of Bombyx mori. a Total protein concentration of midgut tissues of $B$. mori infected with $N$. bombycis and uninfected. $\mathbf{b}$ The relative content of fatty acid in infected and uninfected midgut tissue of $B$. mori; $\mathrm{C}_{17} \mathrm{H}_{34} \mathrm{O}_{2}$ : hexadecanoic acid methyl esters; $\mathrm{C}_{18} \mathrm{H}_{28} \mathrm{O}_{3}$ : benzenepropanoic acid, 3,5-bis(1,1-dimethylethyl)-4-hydroxy-, methyl ester); $\mathrm{C}_{18} \mathrm{H}_{36} \mathrm{O}_{2}$ : palmitic acid ethyl ester; $\mathrm{C}_{19} \mathrm{H}_{38} \mathrm{O}_{2}$ : methyl stearate; $\mathrm{C}_{20} \mathrm{H}_{40} \mathrm{O}_{2}$ : octadecanoic acid ethyl ester; c The content of ATP in infected and uninfected midgut tissue of $B$. mori. Data are presented as the mean \pm SE of triplicate experiments. Statistically significant differences between the infected groups and control non-infected groups were determined using Student's t-test. *P-value of $\leq 0.05$ was considered as statistically significant

proteases are involved in protein degradation. Some serine protease genes were also identified in our subtractive library. Fortunately, the molecular evidence was also eventually supported by the biochemical data that the protein content of infected midgut tissue was significantly decreased compared with the uninfected midgut. In addition, fat degradation related genes in $B$. mori were also significantly upregulated due to $N$. bombycis invasion, including lipase-1 (NP_001036966), fatty acylcoenzyme A dehydrogenase (NP_001086623.1), and acetyl coenzyme A thiolase (NP_001093296.1). Among these, the expression level of lipase-1 was particularly high (Fig. 4), indicating that $N$. bombycis infection accelerated fat hydrolysis and fatty acid $\beta$-oxidation in $B$. mori midgut cells. Moreover, because $N$. bombycis lacks the pathways for de novo synthesis of fatty acids but retains a fatty acid transporter gene and long-chain fatty acid CoA ligase gene [6], we speculate that $N$. bombycis could obtain short-chain fatty acids from the host cells to synthesize long-chain fatty acids. This would result in significant changes in fatty acids content in the host midgut. Unfortunately, further GC-MS analysis showed that the fatty acids content of infected midgut did not change significantly compared with the uninfected groups. Based on this result, we speculate that $N$. bombycis prefer obtaining proteins over fatty acids from the host to ensure the material requirements for parasitic life.

Given that $N$. bombycis infection leads to elevated expression of host proteins, fat degradation and ATP synthesis related gene, and a significant decline of protein and ATP content in host midgut, we believe that the amino acids and ATP needed for $N$. bombycis growth and reproduction are subtracted from the host, rather than being synthetized de novo. The advantage of subtracting amino acids and ATP from the host is that $N$. bombycis can quickly acquire large amounts of materials and energy required for its life-cycle. However, the disadvantage of this strategy is also apparent: as the extended progression of the infection duration, the catabolism rate of proteins and ATP is about to surpass their synthesis rate, which would result in the damage and eventual death of the host cells. Therefore, keeping an appropriate rate for the utilization of proteins and ATP from the host cells is important for the parasitic life of $N$. bombycis.

\section{Conclusions}

$\mathrm{SSH}, \mathrm{qPCR}$, and ATP, protein and fatty acids content analysis suggest that $N$. bombycis invasion can activate host protein degradation and potentially accelerate host ATP production, to ensure enough nutrients and energy for the completion of the parasite life-cycle. This study enriches our understanding of how the microsporidia $N$. bombycis obtain energy and material from host cells, and provides valuable information for future functional investigations of key molecules responsible for energy and material transfer from $B$. mori to $N$. bombycis, to use as a basis for the identification of drug target candidates.

\section{Additional files}

Additional file 1: Table S1. The differently expressed genes in the SSH CDNA library. A total of 110 differentially expressed genes in the midgut of Bombyx mori during the early stage of Nosema bombycis infection were systemically identified by suppression subtractive hybridization (SSH). (DOCX 29 kb)

Additional file 2: Table S2. The relative content of fatty acids in control and infection midgut tissue of Bombyx mori. Fatty acid quantitative data for midgut tissue of B. mori. Results expressed in area \% of fatty acid in the sample. (DOCX $71 \mathrm{~kb})$ 


\section{Abbreviations}

BmNPV: Bombyx mori nucleopolyhedro virus; CPV: cytoplasmic polyhedrosis virus; ESTs: expressed sequence tags; GO: Gene Ontology; KEGG: Kyoto Encyclopedia of Genes and Genomes; Ip: lipase; mp: membrane protein; qPCR: quantitative real-time PCR; $s p 1$ : serine protease 1; sp11: serine protease 11; sp5: serine protease 5; SSH: suppression subtractive hybridization; TCA: tricarboxylic acid; UPS: ubiquitin-proteasome system; vas: vacuolar ATP synthase subunit $G$

\section{Acknowledgements}

The authors will like to thank Dr Haoxin Zhang and Youjin Hao who given professional writing advice, and other colleagues in our laboratory who contributed to this study.

\section{Funding}

This research was supported by many projects in China. LLY received funding from the National Science and Technology Plan Subject of China (863 Program, 2012AA101301) and the People's Livelihood Science and Technology Innovation Projects of Chongqing (cstc2015shmszx80031), ZL received funding from the Science and Technology Project of the Chongqing Municipal Education Commission of China (KJ1703051) and the Natural Science Foundation of China (31302037) and the Natural Science Foundation Project of Chongqing Normal University of China (13XLB009).

\section{Availability of data and materials}

All data generated or analysed during this study are included in this published article and the supplementary information files.

\section{Authors' contributions}

ZL, LLW and ZZY conceived and designed the study. ZL and YW supervised and performed materials collection, infection treatment and all laboratory experiments, ZL and LLW performed the bioinformatics analyses and manuscript preparation. LLW and ZZY provided overall study supervision. All authors read and approved the final manuscript.

\section{Ethics approval and consent to participate}

The collection of microsporidia and silkworm specimens during the study was approved by the College of Life Science, Chongqing Normal University. Permission to publish the findings was granted by the National Science and Technology Plan Subject of China.

\section{Consent for publication}

Not applicable.

\section{Competing interests}

The authors declare that they have no competing interests.

\section{Publisher's Note}

Springer Nature remains neutral with regard to jurisdictional claims in published maps and institutional affiliations.

\section{Author details}

'College of Life Sciences, Chongqing Normal University, Chongqing 401331 China. ${ }^{2}$ State Key Laboratory of Silkworm Genome Biology, Southwest University, Chongqing 400716, China.

Received: 9 November 2017 Accepted: 26 February 2018 Published online: 06 March 2018

\section{References}

1. Han MS, Watanabe $\mathrm{H}$. Transovarial transmission of two microsporidia in the silkworm, Bombyx mori, and disease occurrence in the progeny population. J Invertebr Pathol. 1988;51:41-5.

2. Wittner M, Weiss LM. The microsporidia and microsporidiosis. Washington, D.C.: ASM Press; 1999.

3. Weidner E, Byrd W. The microsporidian spore invasion tube. II. Role of calcium in the activation of invasion tube discharge. J Cell Biol. 1982;93:970-5.

4. Keeling P. Five questions about microsporidia. PloS Pathog. 2009:5:e1000489.

5. Katinka MD, Duprat S, Cornillot E, Metenier G, Thomarat F, Prensier G, et al. Genome sequence and gene compaction of the eukaryote parasite Encephalitozoon cuniculi. Nature. 2001;414:450-3.
6. Pan G, Xu J, Li T, Xia Q, Liu SL, Zhang G, et al. Comparative genomics of parasitic silkworm microsporidia reveal an association between genome expansion and host adaptation. BMC Genomics. 2013;14:3082-94.

7. Keeling PJ, Fast NM. Microsporidia: biology and evolution of highly reduced intracellular parasites. Ann Microbiol. 2002;56:93-116.

8. Peyretaillade $\mathrm{E}, \mathrm{El}$ Alaoui $\mathrm{H}$, Diogon M, Polonais $\mathrm{V}$, Parisot N, Biron DG, et al. Extreme reduction and compaction of microsporidian genomes. Res Microbiol. 2011;162:598-606.

9. Goldberg AV, Molik S, Tsaousis AD, Neumann K, Kuhnke G, Delbac F, et al. Localization and functionality of microsporidian iron-sulphur cluster assembly proteins. Nature. 2008;452:624-8.

10. Williams BA, Hirt RP, Lucocq JM, Embley TM. A mitochondrial remnant in the microsporidian Trachipleistophora hominis. Nature. 2002:418:865-9.

11. Cheng TC, Xia QY, Ping-Zhen XU, Tan X, Fang T, Identification XZH. comparative analysis of immune-related genes and signalling pathways in the silkworm, Bombyx mori. Acta. Entomol Sinica. 2009;52:235-45.

12. Wu P, Wang X, Qin GX, Liu T, Jiang YF, Li MW, Guo XJ. Microarray analysis of the gene expression profile in the midgut of silkworm infected with cytoplasmic polyhedrosis virus. Mol Biol Rep. 2011;38:333-41.

13. Cheng T, Lin P, Huang L, Wu Y, Jin S, Liu C, Xia Q. Genome-wide analysis of host responses to four different types of microorganisms in Bombyx mori (Lepidoptera: Bombycidae). J Insect Sci. 2016;16:1-11.

14. Ping W, Li MW, Xiu W, Pan Z, Wang XY, Liu T, et al. Differentially expressed genes in the midgut of silkworm infected with cytoplasmic polyhedrosis virus. Afr J Biotechnol. 2009;8:3711-20.

15. Gao K, Deng XY, Shang MK, Qin GX, Hou CX, Guo XJ. iTRAQ-based quantitative proteomic analysis of midgut in silkworm infected with Bombyx mori cytoplasmic polyhedrosis virus. J Proteomics. 2017;152:300-11.

16. Wang XY, Yu HZ, Xu JP, Zhang SZ, Yu D, Liu MH, Wang LL. Comparative subcellular proteomics analysis of susceptible and near-isogenic resistant Bombyx mori (Lepidoptera) larval midgut response to BmNPV infection. Sci Rep. 2017:7:45690.

17. Yu HZ, Wang XY, Xu JP, Ma Y, Zhang SZ, Yu D, et al. iTRAQ-based quantitative proteomics analysis of molecular mechanisms associated with Bombyx mori (Lepidoptera) larval midgut response to BmNPV in susceptible and near-isogenic strains. J Proteomics. 2017:165:35-50.

18. Huang $L$, Cheng $T, X u P$, Cheng D, Fang $T$, Xia Q. A genome-wide survey for host response of silkworm, Bombyx mori during pathogen Bacillus bombyseptieus infection. PLoS One. 2009;4:e8098.

19. Ma Z, Li C, Pan G, Li Z, Han B, Xu J, et al. Genome-wide transcriptional response of silkworm (Bombyx mori) to infection by the microsporidian Nosema bombycis. PLoS One. 2013:8:e84137.

20. Han L, Chen B, Hu S, Liang X, Lu X, Shao Y. Quantitative proteomic analysis of germination of Nosema bombycis spores under extremely alkaline conditions. Front Microbiol. 2016:7:1459.

21. Xia Q, Zhou Z, Lu C, Cheng D, Dai F, Li B, et al. A draft sequence for the genome of the domesticated silkworm (Bombyx mori). Science. 2004;306:1937-40,

22. Livak KJ, Schmittgen TD. Analysis of relative gene expression data using real-time quantitative $P C R$ and the $2^{-\Delta \Delta C T}$ method. Methods. 2001:25:402-8.

23. Bradford MM. A rapid and sensitive method for quantification of microgram quantities of proteins utilizing the principal of proteins-dye binding. Annal Biochem. 1976;72:248-54.

24. Chen GY, Chiu HH, Lin SW, Tseng YJ, Tsai SJ, Kuo CH. Development and application of a comparative fatty acid analysis method to investigate voriconazole-induced hepatotoxicity. Clin Chim Acta. 2015;1:126-34.

25. Zhang X, Zuo X, Yang B, Li Z, Xue Y, Zhou Y, et al. MicroRNA directly enhances mitochondrial translation during muscle differentiation. Cell. 2014 158:607-19.

26. Yang B, Huang W, Zhang J, Xu Q, Zhu S, Zhang Q, et al. Analysis of gene expression in the midgut of Bombyx mori during the larval molting stage. BMC Genomics. 2016;17:866.

27. Hajdusek $\mathrm{O}$, Sima R, Ayllon N, Jalovecka M, Perner J, de la Fuente J, Kopacek $P$. Interaction of the tick immune system with transmitted pathogens. Front Cell Infect Microbiol. 2013:3:26.

28. Dolgikh W, Senderskiy IV, Pavlova OA, Naumov AM, Beznoussenko GV. Immunolocalization of an alternative respiratory chain in Antonospora (Paranosema) locustae spores: mitosomes retain their role in microsporidial energy metabolism. Eukaryot Cell. 2011:10:588-593.

29. Tsaousis AD, Kunji ER, Goldberg AV, Lucocq JM, Hirt RP, Embley TM. A novel route for ATP acquisition by the remnant mitochondria of Encephalitozoon cuniculi. Nature. 2008;453:553-6. 
30. Berg JM, Tymoczko JL, Stryer L, Biochemistry SL. 5th edn. New York: W.H. Freeman. 2002;

31. Voet D, Voet JG. Biochemistry. 4th ed. Hoboken, NJ: John Wiley \& Sons; 2011.

32. Heinz E, Williams TA, Nakjang S, Noel CJ, Swan DC, Goldberg AV, Harris SR, Weinmaier T, Markert S, Becher D, et al. The genome of the obligate intracellular parasite Trachipleistophora hominis: new insights into microsporidian genome dynamics and reductive evolution. PLoS Pathog. 2012;8:e1002979.

33. Nakjang S, Williams TA, Heinz E, Watson AK, Foster PG, Sendra KM, Heaps SE, Hirt RP, Martin Embley T. Reduction and expansion in microsporidian genome evolution: new insights from comparative genomics. Genome Biol Evol. 2013; 5(12): 2285-2303.

Submit your next manuscript to BioMed Central and we will help you at every step:

- We accept pre-submission inquiries

- Our selector tool helps you to find the most relevant journal

- We provide round the clock customer support

- Convenient online submission

- Thorough peer review

- Inclusion in PubMed and all major indexing services

- Maximum visibility for your research

Submit your manuscript at www.biomedcentral.com/submit
Biomed Central 